\title{
PERTINENCIA DE ESTRATEGIAS DEL SISTEMA EDUCATIVO FINLANDÉS, DIRIGIDAS A MEJORAR LOS RESULTADOS DE APRENDIZAJES DE ESTUDIANTES CON NEE
}

\author{
RELEVANCE OF STRATEGIES OF THE FINNISH \\ EDUCATIONAL SYSTEM, AIMED AT IMPROVING THE \\ LEARNING OUTCOMES OF STUDENTS WITH SEN
}

Fanny Zoraida Macancela-Torres ${ }^{1}$ y Fidela Dora Medina-Litardo ${ }^{2}$

\begin{abstract}
Sistema educativo finlandés, necesidades educativas especiales.
\end{abstract}

Palabras clave

\begin{abstract}
Resumen
El objetivo de esta investigación es determinar la pertinencia de las estrategias del sistema educativo finlandés, a través de una revisión sistemática de la literatura dirigida a mejorar los resultados de aprendizaje de los estudiantes con necesidades educativas especiales NEE. Con la finalidad de alcanzar nuestra meta se llevó a cabo un estudio de investigación sistemática de la literatura, la cual incluyó la revisión de artículos publicados desde el año 2015 hasta el 2020 en revistas arbitradas indexadas a las bases de datos de Scopus, EBSCO, Scielo, Google Académico y Redalyc. Los resultados revelaron que a pesar de que Finlandia tiene un sistema educativo exitoso, uno de los mejores del mundo en relación con la excelencia académica y a inclusión educativa, sin embargo, no hay evidencias concretas que haya sido implementado completamente en otros países. En conclusión, el sistema educativo finlandés tiene ciertas diferencias con el sistema educativo ecuatoriano, de tal manera que para su implementación implicaría cambios estructurales e incluso legales.
\end{abstract}

\section{Códigos JEL: I21}

\footnotetext{
${ }^{1}$ Universidad Espíritu Santo (UEES), (Ecuador).

E-mail fmacancela@uees.edu.ec

${ }^{2}$ Universidad Espíritu Santo (UEES), (Ecuador).

E-mail fimedina@uees.edu.ec
} 


Key words
Finnish
education
system, special
educational
needs nee.

\section{INTRODUCCIÓN}

En términos generales se entiende por inclusión la acción de incluir, sin embargo, al tratarse de personas tenemos que detenernos para analizar profundamente lo que conlleva el proceso de inclusión en las escuelas regulares, en donde directivos, docentes, psicólogos, padres de familia, es decir, todos los que conformamos la comunidad educativa debemos atender la diversidad puesto que, en esa diversidad radica la riqueza de una institución y porque no decirlo de nuestra sociedad. La educación inclusiva comenzó a tomar forma y nos permitió creer que puede ser una realidad, cuando la Declaración de Salamanca (UNESCO, 1994) promulgó la inclusión como el camino más idóneo para educar a todos los estudiantes en el sistema educativo ordinario, independientemente de sus características y necesidades individuales, puesto que en algunos casos las barreras las pone una sociedad que no acepta la diversidad por desconocimiento. Actualmente, los países latinoamericanos han dado pasos hacia la reorganización de sus sistemas educativos, esencialmente, bajo el criterio de la educación inclusiva con el precepto de "Educación para Todos". En este contexto en Ecuador, desde el Plan Decenal (2006) y en la Constitución (2008) se han promulgado legislaciones en defensa de los derechos de estas personas. En las últimas décadas en todos los ámbitos de las políticas del Buen Vivir, el Estado y el Gobierno ecuatoriano han efectuado acciones pertinentes para garantizar estos derechos.

En nuestro país se ha experimentado constantes cambios en la educación, los docentes han enfrentado los diversos retos de una sociedad cada vez más exigente, pero sin lugar a duda uno de los mayores desafíos ha sido la inclusión educativa. Siempre han existido necesidades educativas especiales, tales como problemas y trastornos de aprendizaje asociados o no, a una discapacidad, o cualquier situación particular que impida que el estudiante aprenda como lo hacen los niños y niñas de su misma edad. Pero ahora, se ha incrementado el número de estudiantes que presentan NEE en las escuelas ecuatorianas, motivo que lleva a las autoridades estatales a buscar soluciones, apoyándose de las experiencias de otros países y principalmente de la orientación que brindan entidades como la 
UNESCO y la ONU. La UNESCO por su parte ha sido de gran apoyo para todos los países en tema de inclusión. En la Conferencia Internacional de Educación, Cuadragésima Octava reunión, realizada en Ginebra (2008) cuyo tema central fue "La Educación Inclusiva: el camino hacia el futuro", en el cual se mencionó que la inclusión es un proceso orientado a dirigir y a responder a la diversidad de las necesidades (UNESCO, 2008).

Teniendo en cuenta la importancia de la igualdad de derechos para todos, mencionada anteriormente, se ha analizado el sistema de educativo finlandés desde la perspectiva de la inclusión. La presente investigación pretende analizar la pertinencia de las estrategias del sistema educativo finlandés en los avances en los aprendizajes de los estudiantes NEE. Finlandia consiguió establecer un sistema en el que todos los estudiantes tengan un aprendizaje con excelentes resultados, porque los finlandeses le dan prioridad a la educación. Según Melgarejo (2016) este sistema se caracteriza por tener rigor profesional, desempeño académico y competitividad internacional. Además, ha progresado con el transcurso del tiempo y han realizado un arduo trabajo para lograr una inclusión integral. De acuerdo a las evaluaciones internacionales, Finlandia obtuvo excelentes resultados en las pruebas PISA (2018) posicionándose en el quinto lugar, estas pruebas evalúan tres áreas tales como comprensión lectora, matemática y ciencias (Moreno y Aguilar, 2019).

Tabla 1

Resultados del Programa de Evaluación de Estudiantes Internacionales PISA

\begin{tabular}{ccccc}
\hline Puesto & País & $\begin{array}{c}\text { Comprensión } \\
\text { Lectora }\end{array}$ & Matemática & Ciencias \\
\hline 1 & China & 555 & 591 & 590 \\
2 & Singapur & 549 & 569 & 551 \\
3 & Estonia & 523 & 523 & 530 \\
4 & Canadá & 520 & 512 & 518 \\
5 & Finlandia & 520 & 507 & 522 \\
6 & Irlanda & 518 & 500 & 496 \\
\hline
\end{tabular}

También, ocupa el tercer lugar según el Índice de Movilidad Social de acuerdo con el informe de competitividad (2020), que evalúa los factores que impulsan la productividad, conducen al crecimiento económico y social de un país. Estos factores son determinantes para alcanzar una economía competitiva, sustentable e inclusiva (World Economic Forum, 2020).

Tabla 2

Índice de Movilidad Social

\begin{tabular}{ccc}
\hline Puesto & País & Puntuación del Índice Global \\
\hline 1 & Dinamarca & 85.2 \\
2 & Noruega & 83.6 \\
2 & Finlandia & 83.6 \\
3 & Suecia & 83.5 \\
4 & Islandia & 82.7 \\
5 & Países Bajos & 82.4 \\
\hline
\end{tabular}

Fuente: Adaptado de Base de World Economic Forum, WEF 2020 
Además, Finlandia es un país que posee una sociedad que vive feliz, así lo reporta The World Happiness Report (2019). Por consiguiente, se evidencia que Finlandia pone especial interés en el bienestar que gozan los ciudadanos, la tranquilidad de una economía competitiva, que sumado a las políticas de gobierno que garantizan la equidad y la igualdad para todos son los factores que han conducido a un sistema educativo óptimo, que ha logrado captar la atención de otros países, los mismos que se preguntan acerca de las causas de su éxito (Helliwell, Layard, y Sachs, 2019).

Tabla 3

Los Países más felices

\begin{tabular}{ccc}
\hline Puesto & País & Puntaje \\
\hline 1 & Finlandia & 7,809 \\
2 & Dinamarca & 7,646 \\
3 & Suiza & 7,560 \\
4 & Islandia & 7,504 \\
5 & Noruega & 7,488 \\
\hline
\end{tabular}

Fuente: Adaptado del Informe World Happiness Report 2019

Finlandia ha trabajado para diseñar estructuras educativas que frenen la exclusión y fomenten prácticas pedagógicas que favorezcan la inclusión. Siendo el factor más importante para la calidad de la enseñanza la profesionalización del maestro. Desde el año 2014, Finlandia renovó su plan de estudios nacional para la educación básica y se lo introdujo graduablemente en las escuelas desde el 2016. Este plan lo elaboró la Junta Nacional de Finlandia (Finnish National Agency for Education, 2016) teniendo en cuenta los objetivos nacionales y las necesidades locales, el cual consiste en un aprendizaje interdisciplinario basado en temas "Phenomenon-Based Teaching and Learning", en el cual los estudiantes resuelven problemas de forma individual y grupal (Symeonidis y Schwarz, 2016).

Este sistema educativo está basado en la participación y el entorno favorable para todos. Adoptar todo lo positivo en la educación de calidad de Finlandia, podría servir para mejorar los resultados de aprendizaje de los estudiantes que presentan NEE, pues este sistema nos enseña que la equidad y la excelencia pueden coexistir en la educación. Según Leo Pahkin (2017) Consejero de Políticas Educativas del Ministerio de Educación de Finlandia, en una entrevista realizada por Heike Freire, menciona que el sistema educativo finlandés está basado en cinco principios, tales como, una política educativa común, una visión de futuro, la equidad, la descentralización y por último, una cultura basada en la confianza. Actualmente, Leo Pahkin dirige un equipo de expertos que ayudan a las escuelas a implementar las políticas nacionales principalmente en matemáticas y ciencias. Además, colabora con diversos organismos internacionales y ha impartido conferencias en más de 40 países. Por sus orígenes modestos, es un ejemplo vivo de un sistema basado en la igualdad de oportunidades. Como suelen decir en su país, "no podemos permitirnos desperdiciar ningún cerebro" (Freire, 2018).

Por tal razón, se plantea como objetivo, determinar la pertinencia de las estrategias del sistema educativo finlandés, por medio de una revisión sistemática de la literatura dirigida a mejorar los resultados de aprendizaje de los estudiantes con necesidades educativas especiales NEE. Para cumplir el objetivo de la investigación se propuso un estudio de revisión sistemática de la literatura, la cual incluyó la revisión de artículos publicados desde el año 2015 hasta el 2020 en revistas arbitradas indexadas a las bases de datos de Google Académico, Scopus, EBSCO, Scielo y Redalyc. Para ello se identificaron 
las palabras claves tanto en español como en inglés, a el sistema educativo finlandés (Finnish Educational System); b. necesidades educativas especiales (Special Educational Needs). Se hizo una revisión utilizando los artículos que contenían información relaciona con la pertinencia del sistema educativo finlandés y con las palabras dependientes resultados de aprendizaje en estudiantes con NEE.

\section{REVISIÓN DE LITERATURA}

\section{Necesidades Educativas Especiales NEE}

Desde hace 25 años de la Declaración de Salamanca hasta la época actual, se ha vivenciado momentos de cambios relevantes en las políticas de educación inclusiva en nuestro territorio, pero nuestro sistema educativo aún muestra unas series de falencias e inconformidades en el tema de la inclusión de los estudiantes con Necesidades Especiales (Rosas, Staig, Lazcano, Palacios, Espinoza, Aro \& Imbernón, 2019). Dentro de sus puntos más importantes acerca de Estudiantes con Necesidades Especiales, la Declaración de Salamanca manifiesta que todo niño posee características, intereses, capacidades y necesidades de aprendizaje que son propias de su edad. También que todos los sistemas educativos deben ser diseñados acorde a sus necesidades y los programas aplicados de modo que tengan en cuenta, toda la gama de esas diferentes características y necesidades (UNESCO, 1994).

Además, los estudiantes con NEE deben tener acceso a las escuelas ordinarias, que deberán integrarlos en una pedagogía centrada en el niño, capaz de cubrir sus requerimientos. Las escuelas ordinarias con esta orientación representan el medio más eficaz para combatir las actitudes discriminatorias, crear comunidades de acogida, construir una sociedad justa $y$ lograr la educación para todos. Por consiguiente, proporcionan una educación efectiva para la mayoría de los estudiantes, mejoran la eficiencia y en definitiva, la relación costo-eficacia de todo el sistema educativo. Para la Organización de las
Naciones Unidas para la Educación, la Ciencia y la Cultura la (UNESCO, 1994) la "Declaración de Salamanca y Marco de Acción para las necesidades educativas especiales" fue un avance significativo en la defensa de una educación inclusiva de excelencia para atender la diversidad de los estudiantes y particularmente para las personas con discapacidad. Más adelante se consolida y existen cambios en la Convención de los Derechos de las Personas con Discapacidad que se dio lugar en la Organización de Naciones Unidas (ONU, 2006).

La ONU (2006) manifiesta que se prohíbe que las personas con discapacidad queden excluidas del sistema de educación. También se dispuso que se eliminen disposiciones legislativas donde se establecen limitaciones y que perjudiquen a la correcta inclusión. Tampoco se debe permitir que la exclusión, sea por su deficiencia o grado de dicha deficiencia. Dejando claro, que la inclusión debe ser con todos los ajustes legales y reglamentarios y jamás se debe eludir la responsabilidad de incluir. En la agenda 2030 que es iniciativa de la ONU para el Desarrollo Sostenible donde se establece un Plan de actuación a favor de las personas, el planeta y la prosperidad, donde el punto relevante es fortalecer la paz mundial y donde se propone un plan de acción a favor de las personas, el planeta y la prosperidad, dejando claro la intención de fortalecer la paz universal y el acceso a la justicia (ONU, 2018). Además, la educación de calidad para todos es un punto importante y tiene como meta hasta el 2030 eliminar las desigualdades de género en la educación y asegurar el acceso igualitario a todos los niveles de la enseñanza y la formación profesional para las personas vulnerables, incluidas las personas con discapacidad, los pueblos indígenas y los niños en situaciones de vulnerabilidad (ONU, 2018). 


\section{Sistema Educativo Finlandés enfocado en atender las Necesidades Educativas Especiales}

Históricamente la inclusión educativa como concepto y práctica en contextos escolares comienza a principios de los 80 en los Estados Unidos y en Europa, como una iniciativa focalizada hacia los estudiantes con discapacidad. Previa aprobación de la Conferencia Mundial acerca de Necesidades Educativas Especiales, La Declaración de Salamanca (1994) considera que los sistemas educativos deben plantear y promover la diversidad como fundamento para la inclusión, aunando acciones efectivas para conseguir la tan anhelada "Educación para Todos", factor determinante para cumplir la propuesta de integrar la escolarización (UNESCO, 1994). García-Cabo (2017) señala que la educación inclusiva ofrece al estudiante la posibilidad de desarrollarse intelectualmente y mejorar su aprendizaje; lo prepara para su inserción en una sociedad integradora y no excluyente, con igualdad de derechos para todos.

Moriña y Carballo (2018) señalan que uno de los obstáculos es la falta de información puesto que, los docentes no perciben cuando un estudiante posee una necesidad educativa especial. Mientras que el sistema educativo finlandés presenta una escuela en donde estos estudiantes son incluidos y valorados por sus diferentes capacidades y además reciben la ayuda necesaria para ser capaces de vencer las dificultades, apoyados por una comunidad que los acoge con amor y paciencia. El modelo finlandés es el más idóneo y posee las estrategias pertinentes para ayudar a los docentes en esta noble y loable labor. Sarrión (2017) menciona que la educación finlandesa tiene como objetivo central, brindar igualdad de oportunidades a todos los ciudadanos, siendo uno de los principios básicos de la educación finlandesa que la totalidad de sus ciudadanos debe tener un acceso igualitario a una educación y capacitación de alta calidad, independientemente de su estatus social, deben tener igualdad de oportunidades para acceder a la educación.
Los objetivos de la educación en Finlandia persiguen principalmente conseguir una educación de excelencia, con gran congruencia social, en donde los estudiantes aprendan a pensar por ellos mismos, a ser críticos con lo que aprenden, a ser personas felices y respetuosas, a socializarse con sus amigos y desarrollarse como seres humanos. Los finlandeses consideran esencial la igualdad de los ciudadanos en el acceso al bienestar y el derecho a su libertad individual en un clima de responsabilidad social. El sistema educativo debe ser eficiente, y no solo eficaz (Ministry of Education and Culture, 2016).

El sistema educativo finlandés se basa en estructuras funcionales, en donde su misión principal es eliminar la exclusión, es decir, la marginación de los estudiantes con NEE. Esas estructuras son un sólido cimiento para la inclusión. De igual manera son decisivas las actitudes y aptitudes de los docentes, para garantizar que todos los estudiantes reciban una educación adecuada. Asimismo, las autoridades estatales, públicas y los servicios sociales son fundamentales en la prestación del apoyo que puedan necesitar los educandos y sus familias. Además, centran su atención en las destrezas que cada estudiante posee con la finalidad de potenciar sus habilidades $\mathrm{y}$ alcanzar la excelencia académica. Moreno (2018) hace referencia que en Finlandia el 99\% de la educación es pública, tanto colegios como universidades. La enseñanza es obligatoria desde los 7 hasta los 16 años, puesto que consideran que ya poseen la suficiente madurez intelectual que les permitirá asimilar los conocimientos, aunque un porcentaje de estudiantes asisten a centros de educación preescolar. Esta es una de las características que hace que este sistema educativo sea tan efectivo.

También, durante los primeros seis años tienen un solo docente. Hasta quinto no hay calificaciones numéricas. Los docentes solo tienen 20 estudiantes por aula, con un máximo de 25 , ponen especial atención en los estudiantes que no avanzan, pues su objetivo primordial es que nadie se quede atrás y reciben clases de apoyo según los 
diferentes niveles de necesidad (Moreno, 2018). Además, los profesores deben tener la habilidad de resolver los problemas para lo cual estarán a la vanguardia de las investigaciones en materia de educación (Rebolledo, 2015). Por tal razón a los estudiantes finlandeses se los considera como los más educados y los de peor rendimiento son excelentes estudiantes cuando se les compara con los de otros países. Melgarejo (2016) menciona que el profesor en Finlandia tiene prestigio, como consecuencia existe una gran demanda por parte de los estudiantes que aspiran ingresar al magisterio. Sin embargo, solo un $10 \%$ son admitidos, puesto que se requiere tener un promedio muy alto. Los aspirantes deben poseer sensibilidad social y se toma en cuenta si realizan labores de voluntariado.

Además, deben tener habilidades, tales como capacidad de comunicación y empatía, aptitudes artísticas y tecnológicas, sólidos conocimientos en matemáticas, lectura comprensiva y redacción. Uno de los aspectos relevantes de este modelo de formación docente es el elevado grado de vinculación entre la formación docente y la investigación. Aguinaga (2017) afirma que a formación del profesorado constituye la clave del éxito del sistema educativo en Finlandia y una cultura basada en la confianza en un sistema diseñado para que los estudiantes desde que empiezan alrededor de los seis o siete años transitan con autonomía a lo largo de su vida estudiantil. La Agencia Nacional de Educación (2016) expresa que la autonomía permite un desarrollo del sistema educativo acorde a las necesidades locales específicas sin intereses políticos. Según García Pedraza (2016) para el ejercicio de la docencia, se debe tener el grado de máster universitario, sólo son aceptados los mejores candidatos, en un proceso de selección de elevada exigencia, demostrando dotes y cualidades personales excepcionales. En consecuencia, se renueva el voto de confianza de la sociedad a la docencia, logrando así el respeto de toda la comunidad.

\section{Estrategias finlandesas que permiten la inclusión para los estudiantes con Necesidades Educativas Especiales}

El sistema educativo en Finlandia se ha basado en la filosofía de la inclusión desde hace mucho tiempo. La educación básica es igual para todos. No existe una división por habilidades, sino que los estudiantes son apoyados de forma individual para que puedan completar satisfactoriamente su educación básica (Finnish National Board of Education, 2019). Este sistema se identifica por ser capaz de descubrir cualquier problema de aprendizaje. Desde el preescolar, a los estudiantes les realizan varias pruebas para descubrir si existe algún inconveniente en el proceso. En el supuesto que sean positivos los estudios realizados, los estudiantes ingresan a la primaria en clases especializadas. Sin embargo, solo puede haber cinco estudiantes por establecimiento $y$ tener profesores especializados. Si las dificultades que presenta son imperceptibles se procede a una inclusión total. Para el efecto los niños deben sentirse a gusto para facilitar la transición del hogar a la escuela. Los espacios de trabajo son cómodos, los pasillos están decorados con sus trabajos (Moreno, 2018). En Finlandia los profesores les enseñan dónde y cómo pueden desarrollar las habilidades y destrezas. Después los docentes evalúan las habilidades que han adquirido los estudiantes. En la formación básica hasta los doce años no existen calificaciones ni exámenes (Moreno, 2018). Los niños tienen ideas originales y hacen las cosas de forma distinta a los adultos la tarea del docente es apoyar esa creatividad. Cuentan con docentes auxiliares y un orientador por cada 200 estudiantes para la secundaria. Otra de las estrategias es comprender que un estudiante que es feliz aprende más rápido (World Happiness Report, 2019). Si está cómodo en el aula, se siente libre de aprender a su ritmo, va a aprender más fácil los conocimientos que les son necesarios. Helliwell, Layard y Sachs (2019) afirman que Finlandia es el país más feliz, lo cual demuestra la gran valoración que le otorgan 
al progreso social y al bienestar de las personas (ONU, 2020).

En la propia cultura escolar, esta confianza se manifiesta en una gran autonomía pedagógica para el maestro, cuyo desempeño laboral no está sujeto a un mecanismo de control o supervisión, puesto que reconocen su alto grado de preparación. En Finlandia, si se sospecha que un estudiante no puede terminar el ciclo de educación obligatoria en el plazo previsto de nueve años, a causa de alguna discapacidad o enfermedad, ese estudiante puede comenzar su escolarización un año antes que el resto y tomar dos años de educación preprimaria. Otra opción sería que comience ese plan de educación obligatoria extendida a la edad de cinco años (Finnish National Board of Education, 2004). Este sistema, pone especial atención en aquellos estudiantes que presentan dificultades al momento de comenzar la etapa escolar, de tal manera que la institución toma medidas de acuerdo con las circunstancias para facilitar esa transición. Las medidas van desde comenzar un año antes, modificar el horario de clases, hasta omitir ciertas asignaturas. Además, las evaluaciones se realizan acorde a sus aprendizajes. Una educación especial a media jornada ha demostrado ser muy conveniente para lograr buenos resultados en el aprendizaje (Didriksson, 2017).

Los estudiantes con las discapacidades más graves no estudian asignaturas concretas, sino que, por el contrario, su instrucción se divide en ámbitos funcionales, como la capacidad motriz, el lenguaje y la comunicación, habilidades para la vida en sociedad, actividades de la vida cotidiana y la capacidad cognitiva. Sus progresos se evalúan atendiendo a esos ámbitos. Los niños con retraso mental profundo estudian casi siempre en el marco de sus propios grupos bajo la orientación de maestros y asistentes personales. Cada vez más, esas clases se vinculan con las escuelas regulares (Finnish National Board of Education, 2004). Los resultados satisfactorios de las escuelas finlandesas se basan en el éxito alcanzado por todos los estudiantes. Esos resultados no se han obtenido impartiendo clases a los estudiantes con necesidades especiales y los de aprendizaje más lento en escuelas separadas, sino incorporando a esos estudiantes en las clases y escuelas ordinarias. El rasgo fundamental es una escuela polivalente $y$ equitativa que beneficia a todos los estudiantes por igual, puesto que en Finlandia existen mínimas diferencia entre una escuela y otra, tal vez diferencias regionales más no estructurales. Sahlberg (2015) menciona que en Finlandia no existe una definición legal de NEE, y todo estudiante que se retrase en alguna materia recibe apoyo especial hasta que sea necesario. Así, un 51\% de los estudiantes finlandeses reciben a lo largo de su escolarización, algún tipo de educación especial. Y por ser más del $50 \%$, lo especial deja de serlo.

Según el Ministerio de Educación y Cultura, la Junta Nacional de Educación de Finlandia y el Centro para la movilidad Internacional CIMO (2016) los estudiantes en Finlandia son considerados miembros importantes para su sociedad, por lo que, de acuerdo a la ley, tienen derecho a recibir apoyo total del estado en sus estudios, y se garantizará una "enseñanza personalizada u orientada según sus necesidades" que maximice el potencial de cada individuo. Para los finlandeses el que cada niño tenga tiempo y espacio para desarrollarse es un principio básico, puesto que la educación aprovecha los periodos más relevantes de su desarrollo, incentivando a todos los niños para que razonen, piensen de manera independiente $y$ sean creativos por excelencia. Finnish National Board of Education (2019) afirma que la educación para necesidades especiales de manera general se imparte en conjunción con la convencional ya que, Finlandia tiene como ideología educar de manera prioritaria a los estudiantes con necesidades especiales, para lo cual los integra con el resto del grupo.

Sin embargo, si un estudiante presenta marcadas dificultades de aprendizaje en su grupo regular se lo deriva para que reciba una educación orientada a su necesidad 
especial. Y por lo general siempre, esta enseñanza se imparte en las mismas escuelas a la que el estudiante pertenece. Todos los niños en edad de escolaridad obligatoria tienen derecho a un apoyo general, que consiste en una educación de alta calidad, así como orientación y guía. Según el Ministerio de Educación y Cultura (2016) la educación básica de acuerdo a la ley, busca que la mayoría de las adecuaciones para estudiantes con NEE se den dentro del aula regular para ello existen tres niveles de apoyo para todos los estudiantes que lo requiera, apoyo General, Intensificado o Especial. La educación para necesidades especiales se aplica en situaciones donde hay problemas de aprendizaje lingüístico y matemático, dificultad de aprendizaje en alguna materia específica, habilidades sociales $\mathrm{y}$ de aprendizaje o cuando hay un compromiso en la asistencia escolar del estudiante, en este último caso pasa a ser parte del apoyo intensificado. Dentro de los tres niveles de apoyo existen medidas estándar que se encuentran disponibles para todos los estudiantes. Todos los niveles de apoyo implican procesos de diferenciación, guía, asistencia personal, servicio de bienestar para los estudiantes, psicólogo escolar y orientación estudiantil.

Leo Pahkin (2018) destaca que la educación es inclusiva en todo aspecto ya que lo más importante no son los contenidos, sino que los alumnos tengan conocimiento de que hacer al final de los ciclos escolares. Porque es primordial el proceso de cómo aprenden ya que se identifica una gama de competencias para el siglo XXI que son trasversales, tales como aprender a aprender, cuidado propio y el de los demás, ser capaz de expresarse, de comprender y de manejar unas coordenadas culturales, el multi-alfabetismo que es el manejo de números, figuras, curvas, letras, entre otros y tener el conocimiento para participar en la vida social de manera democrática y sostenible. La transversalidad, la colaboración entre diferentes áreas, y personas es fundamental. Tienen como premisa que, si el estudiante participa activamente preguntando, y que si les interesa resolver problemas se debe facilitar actividades que estén acordes con la vida real $\mathrm{y}$ permitirles que indaguen las soluciones conjuntamente, ya que aprenderán a trabajar en equipo. Leo Pahkin (2018) señala que el sistema educativo finlandés está basado en cinco principios fundamentales, tales como, una política educativa común, es decir que no cambia con los gobiernos de turno; una visión de futuro, puesto que no olvidamos que nuestros estudiantes después de doce años estarán inmersos en la sociedad como entes productivos; la equidad, que resume con la frase célebre "a cada cual según su necesidad, y a cada cual según su capacidad"; la descentralización es decir, cada ayuntamiento es responsable de la educación, nombran a los directivos y elaboran el curriculum acorde a marco común referencial y por último, una cultura basada en la confianza.

Tabla 4

Los principios del Sistema Educativo finlandés según Pahkin

Principios

Principio 1

Principio 2

Principio 3

Principio 4

Principio 5
Descripción

Política educativa para todos los finlandeses.

Visión futurista.

Equidad.

Descentralización.

Cultura basada en la confianza. 


\section{Tabla 5}

Los pilares de la educación según Sahlberg

\begin{tabular}{ll}
\hline Pilares & \multicolumn{1}{c}{ Descripción } \\
\hline Pilar 1 & Igualdad de oportunidades para todos. \\
Pilar 2 & Gratuidad en la Educación. \\
Pilar 3 & Atención temprana a las NEE. \\
Pilar 4 & El curriculum proporciona lineamientos generales y un marco \\
Pilar 5 & Lomún. \\
Pilar 6 & Los docentes son valorados y su jornada es organizada. \\
Pilar 7 & Los estudiantes combinan el estudio con el juego. \\
Pilar 8 & Se premia la curiosidad y la participación. \\
Pilar 9 & Informes descriptivos a los padres de familia. \\
Pilar 10 & Conciliación entre la vida laboral y familiar. \\
\hline
\end{tabular}

Fuente: Elaboración propia

Sahlberg (2015) señala que el éxito del sistema educativo finlandés radica en los diez pilares fundamentales, tales como que la educación es gratuita y accesible a todos. El Sistema Educativo de Finlandia (2016) establece la gratuidad e igualdad y se caracteriza por ofrecer a todos las mismas oportunidades. También, brindan atención temprana a las necesidades educativas especiales. El curriculum proporciona lineamientos generales y un marco común, sin embargo, las escuelas pueden adaptarlo de acuerdo su región. Los fondos públicos se hacen de manera equitativa. El profesorado durante la jornada laboral no solo se dedica a impartir clases sino también realizan trabajo colaborativo, preparan sus lecciones e investigan. Además, los estudiantes dedican tiempo al estudio, pero

\section{Pertinencia del sistema educativo finlandés en otros países}

Según resultados de búsquedas de la web en los diferentes repositorios de la base de datos para investigación, el sistema educativo finlandés no ha sido implementado en su totalidad solo se ha tomado algunas alternativas, técnicas, propuestas, y conversatorios sirve de guía de referencia en países que mencionaremos a continuación: también le dan prioridad al juego. Se premia la curiosidad y la participación. Los informes entregados a los padres de familia no son numéricos sino descriptivos con la finalidad de evitar la preocupación por los promedios. La conciliación de la vida laboral y familiar es trascendental para que haya más involucramiento de los padres de familia. El desarrollo de competencias es fundamental durante el proceso de enseñanza con la finalidad de fomentar y estimular el desarrollo de habilidades en el último año de educación básica y de educación secundaria superior, la práctica laboral a través de contratos de aprendizaje entre el estudiante, la institución educativa y la empresa (Lähdemäki, 2019).

Oinonen y Blanco (2019) demuestran que Argentina analiza la manera en que las políticas públicas de Educación Superior de Argentina y Finlandia contribuyen o no a reducir las desigualdades sociales en sus respectivos sistemas universitarios. Donde se compara las características de los sistemas sociales y las tendencias de las políticas ejecutadas en la Educación Superior en Argentina y Finlandia, mostrando su cercanía o distancia con respecto de los principios que se 
fundamentan en la Educación Superior de cada país. También se estudia el nivel democrático para dar a conocer si las políticas contribuyen a reducir las desigualdades sociales en la Educación Superior. Se demostró mediante esta investigación que las políticas educativas cuando se fundamentan en la lógica del mercado, las desigualdades en la Educación Superior tienden a incrementarse, incluso, en el país nórdico con estado de bienestar. Entonces podemos apreciar que las correctas políticas públicas son esenciales para los avances de la educación en cada país.

López (2019) considera que es relevante para Colombia realizar un análisis de políticas públicas aplicadas y contrastarlas con políticas educativas que han adoptado países como Chile y Finlandia. Algunas instituciones de educación superior en Colombia ya han implementado métodos de educación finlandés en sus programas de educación, y a través de tres empresas finlandesas, Mehackit, FinlandWay y Tuudo, acompañado con el apoyo de ProColombia, buscan en un futuro invertir en la implementación del modelo educativo de Finlandia en territorio colombiano para beneficiar a estudiantes desde la primera infancia hasta la educación superior. Colombia está consciente de lo que implica el cambio del sistema educativo y la importancia que las políticas de estado favorezcan esta transformación. Para lo cual se analiza las políticas educativas de Colombia y Finlandia, se pone especial interés en la financiación y gestión del personal docente, lo cual permitirá establecer diferencias y similitudes entre los modelos educativos de ambos países para facilitar su ejecución.

Abadía, Bernal y Muñoz (2018) señalan que Colombia históricamente se ha ubicado en los últimos lugares de las pruebas PISA, mientras que Chile tiene el mejor desempeño de Latinoamérica y Finlandia ocupa constantemente los primeros lugares en el mundo. Según las Brechas en el Desempeño Escolar en PISA donde se explica la diferencia de Colombia con
Finlandia y Chile, los resultados obtenidos demuestran que, si los estudiantes colombianos tuvieran la distribución de características familiares de los finlandeses o chilenos, su rendimiento sería significativamente mejor que el actual. Dentro de este componente, la variable de mayor impacto para explicar la brecha es la riqueza de los hogares. Adicionalmente, dentro del conjunto de factores analizados, las variables intrínsecas como actitudes y motivación del estudiante frente al aprendizaje cobran importancia para explicar las diferencias en el rendimiento escolar en la comparación con Finlandia, estos los factores escolares en el caso de Chile explican la mayor proporción de la brecha. Los resultados muestran que hay efectos diferenciados a lo largo de la distribución de puntajes de los diferentes determinantes del desempeño.

González (2018) menciona que Chile ha logrado grandes avances en el ámbito de la educación, convirtiéndose en uno de los líderes de la región, presentando los índices de mayor cobertura con un promedio de matrículas del $88 \%$ en niños de preescolar, según Reporte de Economía y Desarrollo (RED, 2016) todavía tiene retos que enfrentar como el desarrollo integral de habilidades de los jóvenes y la culminación de los estudios de la Corporación Andina de Fomento (CAF, 2016). Los resultados alcanzados por Chile durante los últimos años en el tema de educación, se deben a correctas políticas públicas de educación seguidas de leyes que han mejorado sus niveles educativos, los cuales se han basado en modelos de otros países como Finlandia que toma de referencia y aplica el tema de inclusión y la políticas educativas que ha asumido Chile van enfocadas a mejorar la calidad de la educación, así como la equidad y la inclusión con un enfoque de cobertura, pero lo ha adaptado a su entorno.

Villarreal y Abreu (2018) presenta en México los agentes evaluadores aplicados en países alrededor del mundo, su eficacia y operatividad ya que se utilizarán como elementos centrales para establecer una comparativa objetiva de la efectividad en 
los sistemas educativos en Japón, Estonia, Finlandia, Canadá, Corea, y México. Considerando los instrumentos de evaluación que fueron aplicados por medio del Programa para la Evaluación Internacional de Alumnos, el cual tiene como uno de sus propósitos medir el desempeño de estudiantes de 15 años en las tres áreas básicas como son la lectura, la matemática y la ciencia (Gurria, 2016). Además, se pretende establecer un abanico de opciones que México pretende imitar o sencillamente considerar como propuestas para mejorar las prácticas y modelos educativos. Situación que los educadores mexicanos les deja un sólido compromiso de analizar las posibles causas e intentar generar cambios en las prácticas en aras de progresar.

Pérez y Pérez (2015) en cambio pretenden mostrar los resultados obtenidos tras aplicar los estándares y criterios de selección de candidatos que se emplean en Finlandia para admitir a los estudiantes al magisterio en España. La investigación sobre las pruebas de acceso y la selección de candidatos a la formación docente se ha convertido en un campo de estudio muy solicitado en Finlandia, puesto que, en la sociedad finlandesa, la profesión docente siempre ha gozado de un gran respeto y aprecio, lo que ha llevado a que los programas de formación para docentes sean muy comunes y a que los jóvenes con los mejores expedientes en la secundaria soliciten el acceso a las Facultades de Educación. En el año 2007, para responder a la gran demanda de solicitantes, el Ministerio de Educación decidió reformar el sistema de acceso a los estudios de formación docente y añadir un examen nacional a la fase de preselección de candidatos. Demostrando a través de esta investigación que España busca alternativas tras aplicar los estándares y criterios de selección de candidatos que se emplean en Finlandia para admitir a los estudiantes al magisterio, sobre una muestra de alumnos y alumnas de la Facultad de Ciencias de la Educación de Málaga presentando desafíos para conseguir que la actual reforma de acceso a la Universidad en España avance hacia un nivel de mayor calidad.

\section{Comparación del Sistema Educativo entre Finlandia y Ecuador}

El sistema educativo ecuatoriano comparado con el sistema finlandés, presenta marcadas diferencias, lo cual implicaría cambios fundamentales inclusive relacionados al ámbito legal como es el tema del escalafón docente. El escalafón docente es un tema pendiente en el magisterio nacional, puesto desde hace varios años no se lo ejecuta, los títulos, desarrollo profesional, tiempo de servicio y los resultados en los procesos de implementados por el Instituto Nacional de Evaluación no están siendo remunerados adecuadamente como se estipula en la ley (Ministerio de Educación, 2016). En Finlandia, los hijos de empresarios y obreros asisten a la misma escuela mientras que en Ecuador prevalece el poder adquisitivo, es decir si los padres tienen dinero sus hijos estudian en escuelas particulares. También, en este país nórdico se detecta las necesidades educativas especiales oportunamente. Los profesores especializados se encuentran en todos los centros. En nuestro país no se detecta a tiempo las necesidades educativas especiales. El personal docente y administrativos presentan escasa experticia en NEE y los centros especializados son pocos.

Cabe resaltar que los profesores en Finlandia deben poseer un título de cuarto nivel como requisito obligatorio para ejercer la profesión docente incluso en la educación preescolar. En Ecuador los bachilleres pueden ingresar al magisterio y no es requisito imperativo tener una maestría. En Finlandia el docente tiene un valor relevante, la educación es estandarizada y al alcance de todos los ciudadanos. No existe la diferenciación entre los maestros instituciones públicas y privadas, pues todos los docentes son capacitados constantemente. Además, no existen instrumentos de evaluación regulatorios ya que se tiene a confianza y certeza en su preparación académica, tanto es así que para desempeñar la cátedra del nivel de escolaridad es indispensable tener una maestría. Lo que conlleva que los 
docentes tengan un al excelente desempeño profesional, porque cuentan con las capacidades necesarias para planificar sus clases y diseñar sus propios instrumentos de evaluación. Se ha considerado tres aspectos para realizar una comparación entre el Sistema Educativo de ambos países, tales como legal, socioeconómico y pedagógicoinclusivo.
En el Aspecto Legal existen marcadas diferencias entre ambos sistemas. Mientras que en Finlandia las leyes se aplican de acuerdo con la Constitución y la normativa vigente, en Ecuador las leyes constan en la normativa vigente pero no se las ejecuta en función del bien común.

Tabla 6

Aspecto Legal del Sistema Educativo entre Finlandia y Ecuador

Finlandia

La educación finlandesa es gratuita desde la educación básica hasta el grado de maestría. Son provistas de todo el material necesario inclusive se preocupan por la alimentación (Constitución de Finlandia, 2000).

El grado de maestría se ha convertido en el requisito básico y obligatorio de un profesor para poder enseñar en las escuelas finlandesas, incluso para la educación preescolar (Ministry of Education and Culture, 2016).

Selección rigurosa, los profesores de primaria deben tener experiencia como ayudantes durante tres años, los profesores especializados en una materia, deben obtener una maestría en una disciplina y estudiar pedagogía durante uno o dos años (Ministry of Education and Culture, Finnish National Board of Education and CIMO, 2016).
De acuerdo con los artículos 28 y 348 de la ley dispone gratuidad en la educación en todos los niveles, pero se compra materiales didácticos. La alimentación solo se provee colación escolar a escuelas públicas. La educación pública será universal y laica en todos sus niveles, y gratuita hasta el tercer nivel de educación superior inclusive (Constitución de la República del Ecuador, 2008).

Conforme al art. 111 de la LOEI en Ecuador ejercen la profesión docente, bachilleres, previo a un concurso de Méritos y Oposición Quiero ser maestro. No es requisito obligatorio un título de cuarto nivel (Ministerio de Educación, 2016).

Acorde al art. 111 de la LOE requisitos son participar en proceso de Méritos y Oposición, se ingresa sin experiencia, ni título en educación y no es obligatorio tener maestría en educación, es decir no hay rigor en la selección (Ministerio de Educación, 2016). 
Tabla 7

Aspecto Socioeconómico del Sistema Educativo entre Finlandia y Ecuador

\begin{abstract}
Finlandia
El modelo educativo de Finlandia se centra en cinco puntos clave que son la figura del profesor, el método educativo, los centros educativos, la cultura educativa y la política en materia de educación (Informe PISA, 2018). (Organization for Economic Cooperation and Development, 2018). La moderna economía finlandesa da prioridad a la educación Índice de Competitividad Global ICG (Global Economic Forum, 2019).
\end{abstract}

Finlandia se caracteriza por el buen funcionamiento de sus instituciones públicas, su transparencia, su capacidad de innovación, su buen sistema de salud y, en especial, su extraordinario sistema educativo. Según Índice de Competitividad Global ICG (Global Economic Forum, 2019).

En todas las escuelas finlandesas, los hijos de empresarios y obreros estudian juntos.

Los profesores son altamente valorados en Finlandia (Melgarejo, 2016). El profesorado tiene a su disposición un abanico de material listo para usar en sus aulas, las mismas que son confortables y equipadas con material multimedia (Moreno, 2018)

Los niños finlandeses pueden pasar con sus padres sus primeros años de vida. La entrega de la caja de cartón con artículos para recién nacidos es una de las exitosas políticas sociales del país (Instituto de Seguridad Social, 2020), (Maternity Package, 2020) (Kela, 2020).
Ecuador

La economía ecuatoriana no prioriza la educación en los actuales momentos. Se evidencia educción del presupuesto educativo (Ministerio de Economía y Finanzas del Ecuador, 2020).

Ecuador se caracteriza por un funcionamiento inadecuado y una mala gestión de sus instituciones públicas. La educación carece de transparencia e innovación eficaz (Rebotier, 2016).

Prevalece el poder adquisitivo, es decir si los padres tienen dinero sus hijos estudian en escuelas particulares (Instituto Nacional de Estadísticas y Censos, 2016).

Es evidente la desvalorización docente, no se valora el nivel educativo, ni el escalafón docente (Ministerio de Educación, 2016).

Según el art. 152 de la ley dispone que las afiliadas tienen derecho a 2 horas de permiso por lactancia, durante los 12 meses posteriores al parto (Ministerio de Trabajo del Ecuador, 2012). 
Tabla 8

Aspecto Pedagógico-Inclusivo del Sistema Educativo entre Finlandia y Ecuador

\section{Finlandia}

Los estudiantes se incorporan a la educación obligatoria o básica a la edad de siete años, la cual tiene una duración de nueve años (Moreno, 2018).

En las escuelas de Finlandia, los niños tienen tiempo para ser niños y compartir más durante los primeros años con su familia (Moreno y Aguilar, 2019).

Los alumnos finlandeses no tienen que preocuparse tanto de los exámenes. La escuela primaria es prácticamente una época libre de evaluaciones (Moreno, 2018).

Finlandia no existe una definición legal de NEE, y todo estudiante que se retrase en alguna materia recibe apoyos especiales hasta que sea necesario (Sahlberg, 2015).

Se da mayor atención a los estudiantes que presentan dificultades en el aprendizaje y para ellos cuenta con profesores especializados en todos los centros (Didriksson, 2017).

El número de alumnos por clase no supera los 25 (Moreno, 2018).

Finlandia sigue innovando en materia de educación. El uso de tecnología y métodos alternativos en las escuelas finlandesas son una condición sine qua non (Symeonidis y Schwarz, 2016).

\section{Ecuador}

Según el Acuerdo Ministerial 0042-14 la Educación Inicial o Preescolar es el proceso de acompañamiento al desarrollo integral de niños menores de 5 años (Ministerio de Educación, 2014).

Conforme al Acuerdo Ministerial 024-14 los niños ingresan a la escuela a los 3 años a inicial 1, 4 años inicial 2 y 5 años a primero de básica, siendo esta última obligatoria y las otras opcionales (Ministerio de Educación, 2014).

Exámenes son por parciales y quimestrales. La evaluación es permanente, sistemática y científica (Ministerio de Educación, 2016).

De acuerdo a los artículos 185-186 el proceso de evaluación para los estudiantes con necesidades educativas especiales NEE asociadas o no a una discapacidad es el mismo que para el resto de los estudiantes solo se considera el Documento Individual de Adaptación Curricular (Ministerio de Educación, 2016).

No se detecta a tiempo las Necesidades Educativas Especiales. Los profesores no están capacitados y los centros especializados son pocos. No todas las instituciones cuentan con DECE y en la que si hay no realizan seguimiento a los casos (Modelo de Funcionamiento de los Departamentos de Consejería Estudiantil, 2016).

El número de alumnos por clase supera los 38 a 45 . Cuando la ley estipula que no debe superar 25 por aula (Ministerio de Educación, 2012).

Ecuador está en procesos de la innovación educativa. Todavía hace falta mucho por hacer, aunque según el artículo 298 de la (Constitución de la República del Ecuador, 2008) se estable que los gobiernos asignen al sector de la educación, tecnología e innovación entre otros, sin embargo, el acceso a la tecnología a nivel nacional es limitado (Ministerio de Telecomunicaciones y de la Sociedad de la Información, 2016).

Fuente: Elaboración propia

\section{CONCLUSIONES}

En este trabajo de investigación se determinó la pertinencia de las estrategias del sistema educativo finlandés, a través de una revisión sistemática de la literatura dirigida a mejorar los resultados de aprendizaje de los estudiantes con necesidades educativas especiales NEE. Lo más significativo de nuestra investigación fue hacer una comparación entre el sistema educativo finlandés y ecuatoriano porque esto nos permitirá mejorar. De acuerdo con la revisión que se ha realizado no existe evidencia de que se haya implementado el modelo educativo finlandés en su totalidad en otros países, solo se han acogido diferentes acciones que están relacionadas, inspiradas, guiadas y tomados de referencias en determinadas políticas y formas de acceso para mejorar la educación en los países que están buscando 
alternativas de mejoras continuas en sus sistemas educativos.

No se puede implementar totalmente porque existen distintas realidades. El contexto de aprendizaje en Finlandia contrasta con el de Ecuador donde el currículo está constantemente cambiando, dependiendo del gobierno de turno. Las prácticas de educativas serán efectivas si se incorporara políticas o estrategias que mejoren las condiciones de las escuelas, las condiciones socioeconómicas de los hogares, estas medidas deben ser específicas como la alimentación y la dotación de material de estudio. El sistema educativo ecuatoriano con el sistema educativo finlandés tiene diferencias considerables. Para su implementación implicaría ajustar las metodologías y los contenidos al contexto nacional finlandés. Estas buenas prácticas podrían mejorar la motivación y actitud de los ecuatorianos frente al aprendizaje, aspecto en el cual el país presenta grandes desventajas. El Sistema educativo finlandés se caracteriza por la gratuidad y la equidad para todos, además posee un aspecto relevante, que antes que los niños aprendan las tablas de multiplicar, sencillamente aprenden a cómo ser niños, cómo jugar unos con otros y cómo sanar heridas emocionales lo que favorece el desarrollo de distintas habilidades sociales que más adelante son transmisibles al ámbito académico y formativo.

\section{Fortalezas y Limitaciones}

Como una de las fortalezas de nuestra investigación se puede mencionar que existen suficientes bases teóricas, que diversos autores han venido desarrollando a lo largo de los años. Gracias a los excelentes aportes de expertos en el tema de la Educción en Finlandia hemos podido recabar suficiente información que ha sido valiosa para nuestra tesis. Una de las limitaciones es el tiempo destinado para la investigación, que ha sido de cinco meses.

\section{Futuras Líneas de Investigación}

La presente investigación sirve de base teórica para futuras investigaciones que podrían enfocarse en la aplicabilidad de las estrategias en alguna institución educativa del país, lo cual permitirá verificar con datos estadísticos su efectividad en nuestro contexto. Podrían analizar las políticas públicas, sociales, económicas, educativa, entre otras, que vayan en relación con el crecimiento del país. La inclusión oportuna es un contexto democrático, el acceso al conocimiento sin limitaciones de ninguna índole. La igualdad o equidad de oportunidades donde se canalice y viabilice el desarrollo de las competencias requeridas para desenvolverse en sociedad.

\section{REFERENCIAS}

Abadía, L., Bernal, G. y Muñoz, S. (2018). Brechas en el desempeño escolar en PISA: $;$ Qué explica la diferencia de Colombia con Finlandia y Chile. Archivos Analíticos de Políticas Educativas, 26(82).

Aguinaga, S. (2017). ¿Qué podemos aprender del sistema educativo finlandés? La formación del profesorado?

CAF. (15 de Diciembre de 2016). Reporte de Economía y Desarrollo, Más habilidades para eltrabajo y la vida: los aportes de la familia, la escuela, el entorno y el mundo laboral. Caracas, Venezuela: La Corporación Andina de Fomento CAF. Obtenido de https://www.caf.com/es/actualidad/noticias /2016/10/caf-presento-el-reporte-deeconomia-y-desarrollo-red-2016/ Constitución de Finlandia. (1 de Marzo de 2000). Ministerio de Justicia. 2-5. Helsinki, Finalndia.

Constitución de la República del Ecuador. (20 de Octubre de 2008). Registro Oficial 449. Quito, Ecuador: Asamblea Nacional Constituyente.

Didriksson, A. (2017). El cambio educativo en Finlandia; Qué puede aprender el mundo?, de Pasi Sahlberg. Perfiles Educativos, $\quad 39(157), \quad$ 80-158. doi:https://doi.org/10.22201/iisue.2448616 7e.2017.157.58467

Finnish National Agency for Education. (2016). Finnish National Agency for Education. Obtenido de https://www.oph.fi/en

Finnish National Board of Education. (2004). National Core Curriculum for Basic 
Education. Helsinki. Obtenido de https://www.oph.fi/en

Finnish National Board of Education. (2019). National Core Curriculum for Basic Education. Helsinki. Obtenido de https://www.oph.fi/en

Freire, H. (Noviembre de 2018). El Secreto del Sistema Educativo Finlandés. El Mundo de la Educación(8), 26-31.

García-Cabo, M. (2017). Educación Inclusiva: Síndrome de Down, uno más en el aula de Educación Primaria. Pesaguero, Potes.

Global Economic Forum. (2019). Índice de Competitividad Global. Davos, Suiza. Obtenido de https://www.weforum.org/ González, P. (2018). Sistemas educativos basados en políticas públicas, caso Finlandia, Chile y Colombia. Lecciones para Colombia desde la óptica del Derecho Comparado.

Gurria, Á. (2016). PISA 2015 results in focus. PISA in Focus, 67(1).

Helliwell, J. Layard, R. y Sachs, J. (2019). World Happiness Report. New York: ONU. Obtenido de https://worldhappiness.report/ Instituto de Seguridad Social. (Diciembre de 2020). InfoFinand. Recuperado el 2020, de https://www.infofinland.fi/es/vida-enfinlandia/adaptarse-a-finlandia/seguridadsocial-finlandesa

Instituto Nacional de Estadísticas y Censos. (2016). Censo de Población. Quito. Obtenido de https://www.gob.ec/inec

Lähdemäki, J. (2019). Case Study: The Finnish National Curriculum 2016-A Cocreated National Education Policy. En Sustainability, Human Well-Being, and the Future of Education (págs. 397-422). Creative Commons Attribution 4.0 International. doi:10.1007/978-3-31978580-6_13

López, J. (2019). La política pública de educación en Colombia y Finlandia: elementos para la construcción de una educación pública en época de Reformas Educativas Globales. Papel Politico, 24(1), 1-25.

Melgarejo, X. (2016). Gracias Finlandia: Qué podemos aprender del sistema educativo de más éxito (Séptima ed.). Barcelona: Plataforma.
Ministerio de Economía y Finanzas del Ecuador. (2020). Quito. Obtenido de https://www.finanzas.gob.ec/

Ministerio de Educación. (2006). Plan Decenal. Quito. Obtenido de educación.gob.ec

Ministerio de Educación. (2012). Ley de Educación Intercultural Bilingüe LOEI. Obtenido de https://educacion.gob.ec/

Ministerio de Educación. (2014). Acuerdo Ministerial 024-14. Quito. Obtenido de https://educacion.gob.ec/

Ministerio de Educación. (2014). Acuerdo Ministerial 042-14. Quito. Obtenido de https://educacion.gob.ec/

Ministerio de Educación. (2016). Documento de Adaptaciones Curriculares. Obtenido de https://educacion.gob.ec/

Ministerio de Educación. (2016). Instructivo para la aplicación de la evaluación estudiantil. Quito. Obtenido de https://educacion.gob.ec/

Ministerio de Educación. (2016). Ley Organica de Educación Intercultural Bilingüe . Quito. Obtenido de https://educacion.gob.ec/

Ministerio de Educación. (2016). Modelo de Funcionamiento de los Departamentos de Consejería Estudiantil. Quito. Obtenido de https://educacion.gob.ec/wpcontent/uploads/downloads/2017/05/Model o-Funcionamiento-DECEs

Ministerio de Telecomunicaciones y de la Sociedad de la Información. (2016). Obtenido de https://www.telecomunicaciones.gob.ec/ Ministerio de Trabajo del Ecuador. (2012). Código de Trabajo Artículo 152. Quito. Obtenido de http://www.trabajo.gob.ec/ Ministry for Foreign Affairs, Department for Communication. (18 de Diciembre de 2020). This is Finland. Obtenido de https://finland.fi/tag/maternity-package/ Ministry of Education and Culture. (2016). The Finnish Education System. Helsinki, Finlandia. Obtenido de https://minedu.fi/ Ministry of Education and Culture, Finnish National Board of Education and CIMO. (2016). La Educación finlandesa en síntesis. Helsinki.

Moreno, J. y Aguilar, F. (2019). Fundamentos ontológicos del sistema 
educativo finlandés como referente para superarse en contextos emergentes. Sophia, 1(27),

41-48.

doi:https://doi.org/10.17163/soph.n27.2019 .08

Moreno, P. (2018). Cómo aprenden los estudiantes en Finlandia. Cantabria, España: Universidad de Cantabria.

Moriña, A. y. (2018). Profesorado universitario y educación inclusiva: respondiendo a sus necesidades de formación. 22(SPE), 87-95.

Oinonen, E. y Blanco, C. (2019). Higher education policies and question of social (in) equality: cases of Argentina and Finland. Revista de Educación y Derecho., 19, 1-25.

ONU. (Diciembre de 2006). Convención de los Derechos de las Personas con Discapacidad y Protocolo Facultativo. New York: Naciones Unidas.

ONU. (2018). Agenda 2030 y los Objetivos de Desarrollo Sostenible: Una oportunidad para América Latina y el Caribe. Santiago: Naciones Unidas, CEPAL.

ONU. (2020). World Happiness Report. Obtenido de https://worldhappiness.report/ed/2020/ Organization for Economic Co-operation and Development. (2018). Informe PISA. Paris, Francia: OECD. Obtenido de http://www.oecd.org/

Pahkin, L. (Noviembre de 2017). El secreto del Sistema Educativo Finlandés es la confianza en los profesores. Cuadernos de Pedagogía. (H. Freire, Entrevistador, \& L. Pelayo, Editor) México: Cuadernos de Pedagogía.

Pérez, L. y Pérez, Á. (2015). Los Estándares de Finlandia en las pruebas de selección inicial de profesorado en España. Revista Universitaria del profesorado en España, 29(2), 119-141.

Rebolledo, T. (2015). La formación inicial del profesorado de educación primaria y secundaria en Alemania, España, Finlandia, Francia y Reino Unido.Estudio Comparado. Española de Educación Comparada, 25, 129-148. doi:10.5944/reec.25.2015.14787 Rebotier, J. (2016). El riesgo y su gestión en Ecuador: una mirada de geografía social y política.
Rosas, R., Staig, J., Lazcano, G., Palacios, R., Espinoza, V., Aro, M. \& Imbernón, C. (2019). ¿ Qué Podemos Aprender de los Sistemas Educativos de Chile, España y Finlandia en el Marco de la Declaración de Salamanca? Revista latinoamericana de educación inclusiva, 13(2), 57-74.

Rubén, G. P. (2016). Comprensividad y diversidad en la educación secundaria de Suecia, Finlandia, Reino Unido, Alemania, y España.

Sahlberg, P. (2015). Un sistema escolar modelo. Finlandia demuestra que la equidad y la excelencia pueden coexistir en la educación. Revista de Investigación Educacional Latinoamericana, 52(1), 136145.

doi:https://doi.org/10.22201/iisue.2448616 7e.2017.157.58467

Sarrión, E. (2017). Estudio del sistema educativo finlandés. Publicaciones Didácticas.

Symeonidis, V. y Schwarz, J.F. (2016). Phenomenon-based teaching and learning through the pedagogical lenses of phenomenology: The recent curriculum reform in Finland. University of Lower Silesia, 28(2(56)), 31-47.

The Social Insurance Institution of Finland. (Diciembre de 2020). Kela. Obtenido de https://www.kela.fi/web/en/maternitypacka ge

UNESCO. (1994). Declaración de Salamanca. Marco de acción para las necesidades educativas especiales. Salamanca.

UNESCO. (2008). Conferencia Internacional de Educación, Cuadragésima Octava Reunión. Ginebra.

Villarreal, C. y Abreu, J. (2018). Sistemas Educativos Líderes a Nivel Mundial, su Desempeño, Metodología y Rangos Aprobatorios. Revista Daena, 13(1), 97112.

World Economic Forum. (2020). Índice de Movilidad Social. Davos, Suiza. Obtenido de www.weforum.org 